\title{
X-ray line from the dark transition electric dipole
}

\author{
Chao-Qiang Geng, Da Huang and Lu-Hsing Tsai \\ Chongqing University of Posts \& Telecommunications, \\ Chongqing, 400065 China \\ Department of Physics, National Tsing Hua University, \\ Hsinchu, Taiwan \\ Physics Division, National Center for Theoretical Sciences, \\ Hsinchu, Taiwan \\ E-mail: geng@phys.nthu.edu.tw, dahuang@phys.nthu.edu.tw, \\ lhtsai@phys.nthu.edu.tw
}

ABSTRACT: We study a two-component dark matter (DM) model in which the two Majorana fermionic DM components with nearly degenerate masses are stabilized by an $Z_{2}$ symmetry and interact with the right-handed muon and tau only via real Yukawa couplings, together with an additional $Z_{2}$-odd singly-charged scalar. In this setup, the decay from the heavy DM to the lighter one via the transition electric dipole yields the $3.55 \mathrm{keV}$ $\mathrm{X}$-ray signal observed recently. The Yukawa couplings in the dark sector are assumed to be hierarchical, so that the observed DM relic abundance can be achieved with the leading s-wave amplitudes without a fine-tuning. We also consider the constraints from flavor physics, DM direct detections and collider searches, respectively.

KEYwords: Beyond Standard Model, Cosmology of Theories beyond the SM

ARXIV EPRINT: 1406.6481 


\section{Contents}

1 Introduction 1

2 The model 2

3 X-ray line 3

4 Dark matter relic abundance $\quad 4$

5 Complementary constraints on the model 5

$\begin{array}{lll}5.1 & \text { Flavor physics } & 6\end{array}$

$\begin{array}{lll}5.2 & \text { Direct dark matter detections } & 7\end{array}$

5.3 Collider searches 9

$\begin{array}{llr}6 & \text { Final results } & 9\end{array}$

$\begin{array}{lll}7 & \text { Conclusions and discussions } & 10\end{array}$

\section{Introduction}

Dark matter (DM), although there are overwhelming evidences of its existence from astrophysics and cosmology, is still mysterious from the particle physics point of view and is one of few indications of physics beyond the Standard Model (SM) [1]. Traditionally, one hopes to find some of its properties either by observing nuclear recoils in the underground direct detection experiments [2-4] or by the indirect searches via the measurements of cosmic and gamma rays. ${ }^{1}$ The most recent possible signal comes from the discovery of the unidentified X-ray line at about $3.55 \mathrm{keV}$ from the observation of the Andromeda galaxy and many galaxy clusters, including the Perseus galaxy cluster by XMM-Newton X-ray Space observatory $[6,7]$, which have inspired particle physicists to propose many interesting DM candidates [6-52].

In this paper, we use a simple two-component $\mathrm{DM}_{\text {model }}^{2}$ [54-60] to explain the $3.55 \mathrm{keV}$ X-ray line. The dark sector contains two Majorana fermionic DM fields and a heavy singly-charged scalar which enables the DMs to couple to the right-handed $\mu$ and $\tau$ leptons. Both the DM and singly-charged scalar fields are charged under a $Z_{2}$ symmetry to stabilize the two DM particles. The decay of the heavier DM particle to its lighter sister can only proceed via the dark transition electric dipole moment (EDM), resulting in the observed X-ray signal and guaranteeing the stability of the heavy DM within the Universe age. Furthermore, because of the nearly degenerate DM masses and the particular

\footnotetext{
${ }^{1}$ For a recent review of indirect searches of dark matter, see e.g., [5].

${ }^{2}$ For some recent discussion of multicomponent dark matter models, see e.g., [53].
} 


\begin{tabular}{|c|ccc|}
\hline & $\mathrm{SU}(2)_{L}$ & $\mathrm{U}(1)_{Y}$ & $Z_{2}$ \\
\hline$\ell_{R}$ & $\mathbf{1}$ & -2 & + \\
$\chi_{1,2}$ & $\mathbf{1}$ & 0 & - \\
$\eta$ & $\mathbf{1}$ & -2 & - \\
\hline
\end{tabular}

Table 1. Quantum Numbers for Relevant Fields.

choice of the Yukawa structure, the correct DM relic abundance is achieved via the thermal production by the coannihilation of the two DM particles into $\mu^{ \pm} \tau^{\mp}$ pairs in the $s$-wave with the almost one half for each component.

The paper is organized as follows. In section 2, we show the relevant Lagrangian of the DM sector. We then study the X-ray line in section 3 and the DM relic abundance in section 4 . In section 5 , we consider constraints on the model from flavor physics, DM direct detections and collider searches. In section 6 , we present our numerical results. Our conclusions and discussions are given in section 7 .

\section{The model}

In our model, in addition to the SM particle content, we introduce two Majorana fermionic DM fields $\chi_{1,2}$, which are neutral under the SM gauge groups, and a heavy singly-charged scalar mediator $\eta$ to allow the two DM fields to interact with the right-handed leptons. For simplicity, we restrict ourselves to the case where the two DMs only couple to the two heavier families of leptons, $\mu$ and $\tau$. Both $\chi_{1,2}$ and $\eta$ are odd under the dark $Z_{2}$ symmetry which stabilizes the DM particles. The quantum numbers of the relevant fields are summarized in table 1 . With the assigned quantum numbers for the new particles, we can write down the general renormalizable Lagrangian related to the dark sector as follows:

$$
\begin{aligned}
& \mathcal{L}_{\mathrm{DM}}=\sum_{i=1,2} \bar{\chi}_{i} \not \partial \chi_{i}-\frac{1}{2} \sum_{i, j=1,2} \bar{\chi}_{i}^{c} M_{i j} \chi_{j}+\left(D^{\mu} \eta\right)^{\dagger}\left(D_{\mu} \eta\right)-V(\eta)-\sum_{\substack{i=1,2 \\
\ell=\mu, \tau}}\left(h_{\ell i} \bar{\ell} P_{L} \chi_{i} \eta+\text { h.c. }\right), \\
& V(\eta)=m_{\eta}^{2} \eta^{\dagger} \eta+\frac{\lambda}{2}\left(\eta^{\dagger} \eta\right)^{2}, \quad \text { and } \quad D_{\mu} \eta=\left(\partial_{\mu}-i e A_{\mu}\right) \eta
\end{aligned}
$$

where $M$ denotes the Majorana mass matrix for the two DM fields, $P_{L}=\left(\mathbb{I}-\gamma^{5}\right) / 2$ the usual left-handed projection operator and $A_{\mu}$ the electromagnetic field. We further assume that the Yukawa couplings $h_{\ell i}$ are all real in the bases of the DM and lepton mass states. Furthermore, the constraints from the lepton flavor violating process $\tau \rightarrow \mu \gamma$ and the DM relic density force us to take the hierarchical form of the Yukawa couplings $h_{\mu 1} \sim h_{\tau_{2}} \gg$ $h_{\mu 2} \sim h_{\tau 1}$. For simplicity, we assume the equal value $h_{L}\left(h_{S}\right)$ of the (off-)diagonal entry:

$$
h_{\ell i}=\left(\begin{array}{ll}
h_{L} & h_{S} \\
h_{S} & h_{L}
\end{array}\right), \quad h_{L} \gg h_{S} .
$$



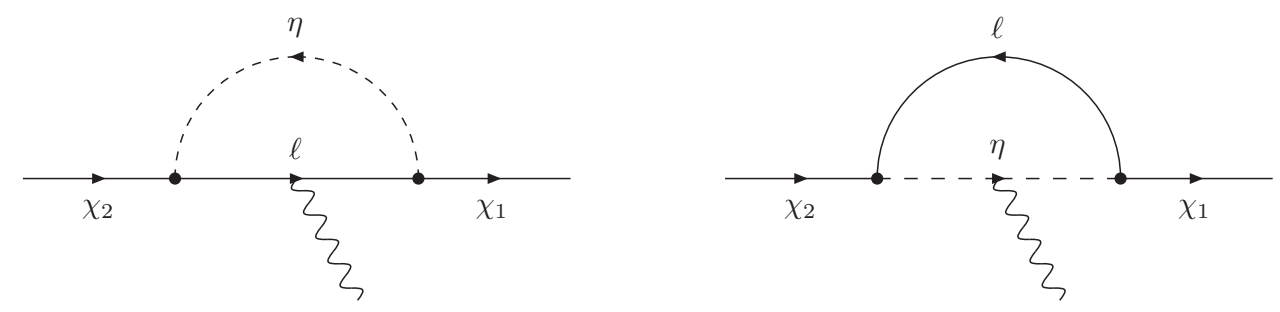

Figure 1. Feynman diagrams for the transition dipole operator between $\chi_{2,1}$.

\section{X-ray line}

It is well-known that the dipole operator of a Majorana particle vanishes. However, for a model with multiple neutral Majorana particles there still exist the transition dipole moments. In this section, we will show that the decay of the heavy Majorana DM particle into the lighter one via the transition electric dipole operator can naturally explain the cluster X-ray line at $3.55 \mathrm{keV}$. We start with the effective dipole operator in our model

$$
\mathcal{O}_{\text {dipole }}=\frac{1}{2} \bar{\chi}_{1} \sigma^{\mu \nu}\left[D_{\chi} \gamma^{5}+i \mu_{\chi} \mathbb{I}\right] \chi_{2} F_{\mu \nu}
$$

where $D_{\chi}$ and $\mu_{\chi}$ denote the dark transition electric dipole moment (EDM) and transition magnetic dipole moment (MDM), respectively. From figure 1, we obtain

$$
D_{\chi}=\frac{e R}{16 \pi^{2}} \frac{\Delta m}{2 m_{\chi}^{2}}\left[1+\frac{m_{\eta}^{2}}{m_{\chi}^{2}} \ln \left(1-\frac{m_{\chi}^{2}}{m_{\eta}^{2}}\right)\right] \approx \frac{e R}{64 \pi^{2}} \frac{\Delta m}{m_{\eta}^{2}}, \quad \mu_{\chi}=0,
$$

where $m_{\chi}(\Delta m)$ stands for the average mass (the mass difference) of the two DM particles and $R=h_{\mu 1} h_{\mu 2}+h_{\tau 1} h_{\tau 2}=2 h_{L} h_{S}$. The decay rate of the heavier DM is then given by

$$
\Gamma=\frac{\Delta m^{3}}{\pi} D_{\chi}^{2} \approx \frac{\Delta m^{3}}{\pi}\left(\frac{e R}{64 \pi^{2}} \frac{\Delta m}{m_{\eta}^{2}}\right)^{2} .
$$

Note that the vanishing value of the transition MDM can be traced back to our assumption of the real Yukawa couplings. However, if this assumption is relaxed, then the MDM would have a nonzero value of $\mu_{\chi}$, given by

$$
\mu_{\chi}=\frac{e I}{16 \pi^{2}} \frac{1}{m_{\chi}}\left[1+\frac{m_{\eta}^{2}}{m_{\chi}^{2}} \ln \left(1-\frac{m_{\chi}^{2}}{m_{\eta}^{2}}\right)\right] \approx \frac{e I}{32 \pi^{2}} \frac{m_{\chi}}{m_{\eta}^{2}},
$$

where $I=\operatorname{Im}\left(h_{\mu 1}^{*} h_{\mu 2}+h_{\tau 1}^{*} h_{\tau 2}\right)$. Clearly, if the imaginary part of the Yukawa couplings is of the same size as the real one, the MDM would be the main contribution since $\mu_{\chi} / D_{\chi} \propto$ $\Delta m / m_{\chi} \sim \mathcal{O}\left(10^{-7}\right)$.

In order to make numerical studies, we need to transform the lifetime presented in ref. [7] into our setup. The prototype model in refs. [6, 7] contains the sterile neutrino with the mass $m_{0}=7.1 \mathrm{keV}$, which decays into an active neutrino plus a photon via the small 
transition magnetic moment with the lifetime in the range $\tau_{0}=0.2 \sim 2 \times 10^{28} \mathrm{~s}$. In our present model, we rescale the above lifetime to match the observed X-ray flux to be

$$
\tau_{\chi}=\frac{\tau_{0} m_{0}}{2 m_{\chi}}
$$

where the factor 2 accounts for the fact that each DM component carries only half of the DM density in the whole Universe and the Galaxy. From eq. (3.5), we see that the predicted lifetime depends on the average DM mass $m_{\chi}$. With eqs. (3.3) and (3.5), we will determine the parameter space in our model to explain the cluster X-ray line.

The measurement of the cluster X-ray line gives some constraint to the mass of the charged scalar $m_{\eta}$. From eqs. (3.3) and (3.5), we can derive the following formula:

$$
m_{\eta}=\left[\frac{\Delta m^{5}}{\pi}\left(\frac{e R}{64 \pi^{2}}\right)^{2}\left(\frac{\tau_{0} m_{0}}{2 m_{\chi}}\right)\right]^{\frac{1}{4}} .
$$

If we fix the values of the dark matter mass and the coupling ratio, for example, $m_{\chi}=$ $10 \mathrm{GeV}$ and $R_{h}=h_{S} / h_{L}=0.1$, it is interesting to see that there is an upper limit on $m_{\eta}$ in the light of the cluster X-ray anomaly and the perturbativity of the Yukawa coupling. If we further take the largest lifetime $\left(\tau_{0}\right)_{\max }=2 \times 10^{28} \mathrm{~s}$ and the maximal value $h_{L}=5$ allowed by the perturbativity, we obtain this upper limit to be $1.86 \mathrm{TeV}$. Yet, this result comes solely from the consideration of the perturbativity and the measured X-ray flux. If we consider more constraints from other experiments, the upper bound on $m_{\eta}$ would further decreased, which will be discussed in section 5 .

\section{Dark matter relic abundance}

It is clear that a successful prediction of the correct DM relic abundance is one of the most important tests for the viability of a DM model. Usually, for a cold DM, the generation of its relic abundance is assumed to proceed by the thermal freezing-out mechanism, which relies on the thermally averaged effective DM annihilation cross section at the time of the freeze-out. Since the two DMs have the highly degenerate masses by the construction, the coannihilations between $\chi_{1}$ and $\chi_{2}$ have to be considered [61].

For a fermionic DM with its freezing-out mainly through $t / u$-channel annihilations, it is well-known that the thermally averaged effective annihilation is dominated by the $p$-wave contribution, which usually requires large couplings and/or light mediators to enhance the annihilation so as not to over-close the Universe. Let us make this point more precise by looking into the leading tree-level Feynman diagrams in figure 2. Since the annihilation with the definite incoming DMs and outgoing leptons involves $t$ - and $u$-channels, if the Yukawa couplings in the two diagrams are the same, the destructive interference between the two channels would cause the leading $s$-wave contribution to be vanishingly small. However, in the presence of the two DM components with the Yukawa structure in eq. (2.2), there is a special process, $\chi_{1} \chi_{2} \rightarrow \mu^{ \pm} \tau^{\mp}$, which does not suffer such a suppression. The $t$ channel diagram is only proportional to $h_{\mu 1} h_{\tau 2}=h_{L}^{2}$, while the corresponding $u$-channel $h_{\tau 1} h_{\mu 2}=h_{S}^{2}$. Based on our construction of $h_{L} \gg h_{S}$, the $t$-channel can not be fully 

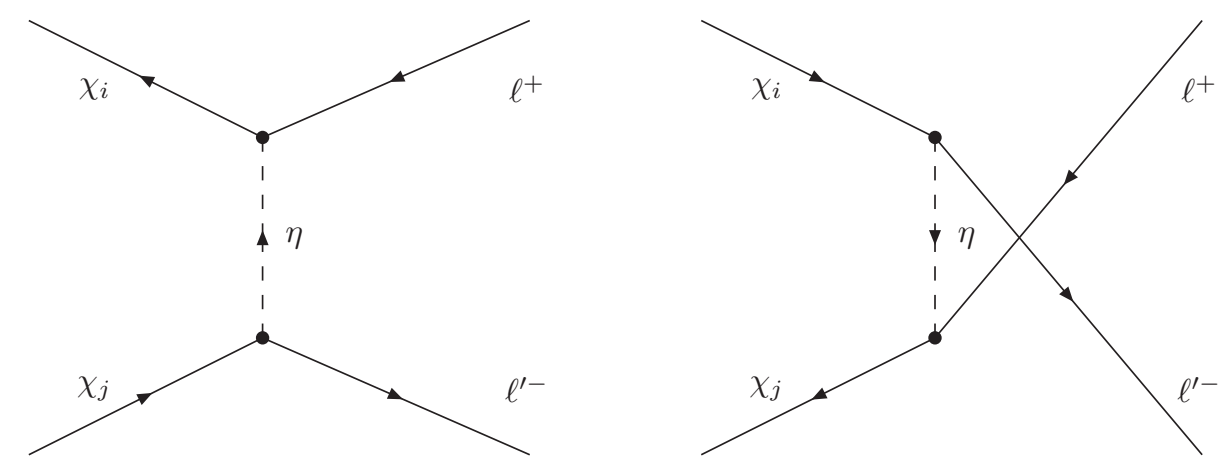

Figure 2. Annihilation diagrams of the DM particles.

cancelled by the corresponding $u$-channel, leading to a large dominant $s$-wave effective cross section:

$$
\left\langle\sigma v_{\mathrm{rel}}\right\rangle=\sigma_{0}=\frac{\left(4 m_{\chi}^{2}-m_{\tau}^{2}\right)^{2}}{512 \pi m_{\chi}^{4}\left(2 m_{\chi}^{2}+2 m_{\eta}^{2}-m_{\tau}^{2}\right)^{2}}\left(h_{L}^{4}\left(4 m_{\chi}^{2}+m_{\tau}^{2}\right)-8 h_{L}^{2} h_{S}^{2} m_{\chi}^{2}+h_{S}^{4}\left(4 m_{\chi}^{2}+m_{\tau}^{2}\right)\right) .
$$

In the following numerical computation of the DM relic abundance, we shall use eq. (4.1) as our effective annihilation cross section. As a result, the analytic solution of the Boltzmann equation describing the evolution of the DM density is given by $[62,63]$ :

$$
\Omega h^{2}=\frac{688 \pi^{5 / 2} T_{\gamma}^{3} x_{f}}{99 \sqrt{5 g_{\star}}\left(H_{0} / h\right)^{2} M_{\mathrm{Pl}}^{3} \sigma_{0}}=\frac{8.7 \times 10^{-11} \mathrm{GeV}^{-2} x_{f}}{\sqrt{g_{\star}} \sigma_{0}},
$$

where the freeze-out temperature $T_{f}$ is implicitly defined in $x_{f}=m_{\chi} / T_{f}$, given by

$$
x_{f}=X-\frac{1}{2} \log X, \quad X=25+\log \left[\frac{g}{\sqrt{g_{\star}\left(T_{f}\right)}} m_{\chi} \sigma_{0} \times 6.4 \times 10^{6} \mathrm{GeV}\right],
$$

and $g_{\star}\left(T_{f}\right)$ denotes the number of relativistic degrees of freedom at $T_{f}$, taken from the tabulated functions in refs. [64, 65]. The final result is shown as the thick red curve in the $m_{\chi}-h_{L}$ plane for several selected benchmark mass and coupling ratios in figure 3 , where we have used the central value of the most recent Planck result $\Omega_{\mathrm{DM}} h^{2}=0.1187 \pm 0.0017$ [66]. Finally, we remark that the neglect of the other annihilation channels to the effective cross sections does not affect the final prediction of the DM relic abundance much, with the correction within $5 \%$ at best, since they are $p$-wave suppressed as mentioned before.

\section{Complementary constraints on the model}

In the previous sections, we have shown that our two-component DM model can not only explain the X-ray line observed from the distant galaxy clusters but also naturally give rise to the desired DM relic abundance in the Universe. However, it is clear that in order for this scenario to be viable, we have to investigate if it satisfies with the constraints from other aspects of particle physics, such as flavor physics, direct DM detections, and collider searches. 


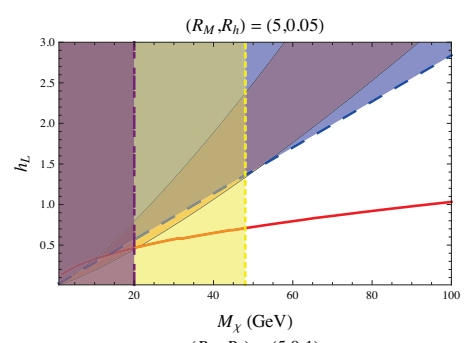

$\left(R_{M}, R_{h}\right)=(5,0.1)$

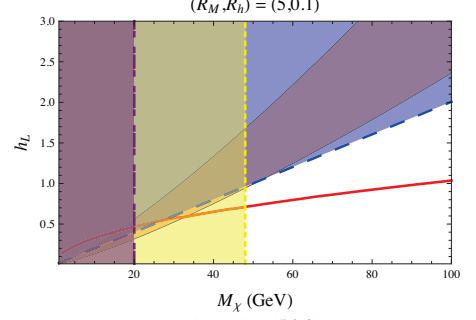

$\left(R_{M}, R_{h}\right)=(5,0.2)$

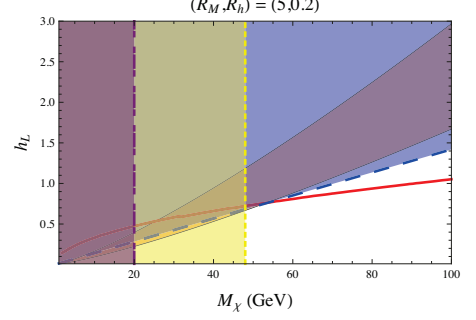

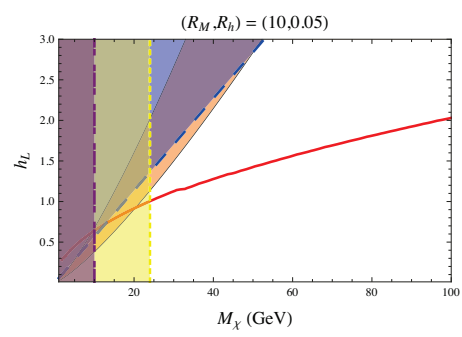

$\left(R_{M}, R_{h}\right)=(10,0.1)$

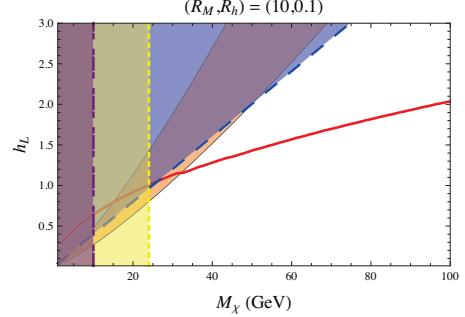

$\left(R_{M}, R_{h}\right)=(10,0.2)$

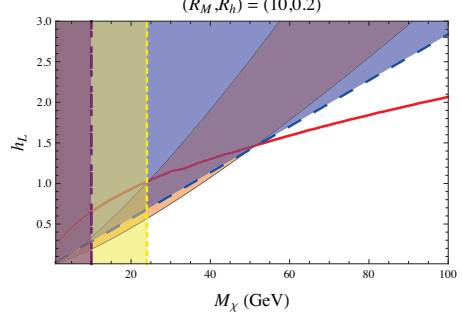

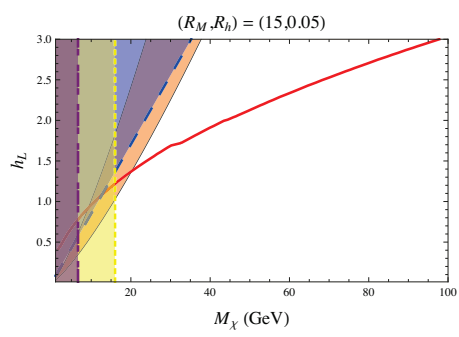

$\left(R_{M}, R_{h}\right)=(15,0.1$

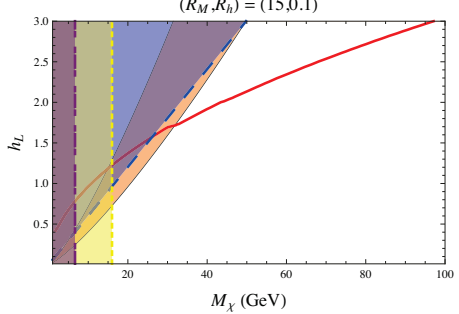

$\left(R_{M}, R_{h}\right)=(15,0.2)$

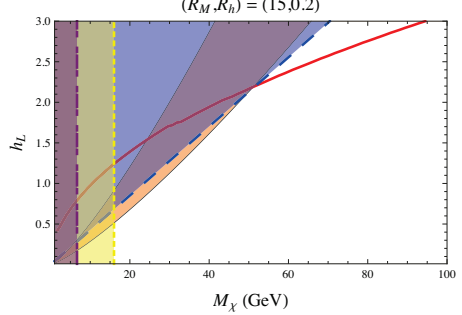

Figure 3. Parameter spaces in the $m_{\chi}-h_{L}$ plane for $R_{M}=m_{\eta} / m_{N}=(5,10,15)$ and $R_{h}=$ $h_{S} / h_{L}=(0.05,0.1,0.2)$, where the thick red curve gives the correct DM relic abundance, the orange region bounded by the thin black curves is accord with the X-ray line observation, and the blue, yellow and purple regions bounded by the dashed, dotted and dot-dashed lines are excluded by the FLV process $\tau \rightarrow \mu \gamma$, LEP and LHC, respectively.

\subsection{Flavor physics}

The most stringent flavor constraint on the model comes mainly from the lepton flavor violation (LFV) process $\tau \rightarrow \mu \gamma$, which proceeds via the dipole operator $\frac{1}{2} \bar{\mu} \sigma^{\mu \nu}\left(A_{L} P_{L}+\right.$ $\left.A_{R} P_{R}\right) \tau F_{\mu \nu}$ with the coefficients $A_{L, R}$ :

$$
A_{L}=\frac{e}{16 \pi^{2}}\left(2 h_{L} h_{S}\right) F_{2}\left(\frac{m_{\chi}^{2}}{m_{\eta}^{2}}\right) \frac{m_{\tau}}{2 m_{\eta}^{2}}, \quad A_{R}=\frac{e}{16 \pi^{2}}\left(2 h_{L} h_{S}\right) F_{2}\left(\frac{m_{\chi}^{2}}{m_{\eta}^{2}}\right) \frac{m_{\mu}}{2 m_{\eta}^{2}},
$$

where the function $F_{2}(x)$ is given by

$$
F_{2}(x)=\frac{1-6 x+3 x^{2}+2 x^{3}-6 x^{2} \log x}{6(1-x)^{4}} .
$$

The branching ratio for this LFV process is [67]:

$$
\mathcal{B}(\tau \rightarrow \mu \gamma)=\frac{3 \alpha_{\mathrm{em}}}{64 \pi G_{F}^{2} m_{\eta}^{4}}\left|\left(2 h_{L} h_{S}\right) F_{2}\left(\frac{m_{\chi}^{2}}{m_{\eta}^{2}}\right)\right|^{2} \mathcal{B}\left(\tau \rightarrow \mu \nu_{\tau} \bar{\nu}_{\mu}\right),
$$

where $\alpha_{\mathrm{em}}=e^{2} /(4 \pi)$ and $G_{F}$ are the fine structure and Fermi constants, respectively. In eq. (5.3), we have neglected the terms proportional to the lepton masses. Using the current 
experimental upper bound of $\mathcal{B}(\tau \rightarrow \mu \gamma)<4.4 \times 10^{-8}$ [68], we present the constrained parameter space as the blue region with the dashed line as the boundary in figure 3 . From the figure, we also see that a lot of parameter space is already excluded by this LFV channel, especially when the hierarchy between $h_{S}$ and $h_{L}$ is not very large, i.e., $h_{S} / h_{L} \sim 0.2$.

\subsection{Direct dark matter detections}

Currently, the most stringent cross section bound for the dark matter mass range of interest is from the LUX experiment [69], in which for the $33 \mathrm{GeV}$ dark matter the spin-independent cross section bound is of $\mathcal{O}\left(10^{-46}\right) \mathrm{cm}^{2}$. For the present leptophilic DM model, the relevant scattering of two DM components with the target nucleus is proceeded by the mediation of a virtual photon. As discussed in the literature [70-76], we first need to identify the relevant effective operators with the DMs coupled to a photon, and then calculate the scattering probability of the DM particles with the target nucleus. Each DM can be viewed as a Majorana particle in the mass eigenstate basis $\chi_{i}$, so that if the scattering does not change the DM component, for the so-called component-conserving scattering, one has to only consider the following two kinds of dimension-six effective operators

$$
\mathcal{O}_{1 i}^{c}=-\bar{\chi}_{i} \gamma^{\mu} \gamma^{5} \partial^{\nu} \chi_{i} F_{\mu \nu}+\text { h.c., } \quad \mathcal{O}_{2 i}^{c}=i \bar{\chi}_{i} \gamma^{\mu} \partial^{\nu} \chi_{i} F^{\alpha \beta} \epsilon_{\mu \nu \alpha \beta}+\text { h.c. },
$$

where $i=1$ and 2. However, as pointed in ref. [70], it can be proved that $\mathcal{O}_{2 i}^{c}=-2 \mathcal{O}_{1 i}^{c}$ by using the Chisholm identity, which results in a single kind of the dimension-six operator for the Majorana fermions. This kind of operators can be matched to the electromagnetic anapole moment of each DM component, which further couples to the current from the target nucleus in the non-relativistic limit and realizes the anapole dark matter [70, 74-76]. However, besides the above component-conserving interactions, there are also transition interactions induced by the real Yukawa couplings,

$$
\mathcal{O}_{1}^{t}=-\overline{\chi_{2}} \gamma^{\mu} \gamma^{5} \partial^{\nu} \chi_{1} F_{\mu \nu}+\text { h.c. }, \quad \mathcal{O}_{2}^{t}=i \overline{\chi_{2}} \gamma^{\mu} \partial^{\nu} \chi_{1} F^{\alpha \beta} \epsilon_{\mu \nu \alpha \beta}+\text { h.c. }
$$

The explicit calculation of the one-loop diagrams in figure 1 leads to the concrete expressions for the Wilson coefficients for these effective operators in the present model [70, 74, 76], given by

- Component-Conserving Couplings:

$$
c_{1 i}^{c}=\frac{-e}{64 \pi^{2} m_{\eta}^{2}} \sum_{\ell} R_{\ell i}^{c}\left[\frac{1}{2}+\frac{2}{3} \ln \left(\frac{m_{\ell}^{2}}{m_{\eta}^{2}}\right)\right], \quad c_{2 i}^{c}=\frac{-e}{64 \pi^{2} m_{\eta}^{2}} \frac{1}{4} \sum_{\ell} R_{\ell i}^{c},
$$

where $R_{\ell i}^{c}=\operatorname{Re}\left(h_{\ell i} h_{\ell i}^{*}\right)$ with $R_{\mu 1(2)}^{c}=R_{\tau 2(1)}^{c}=h_{L(S)}^{2}$, and

- Transition Couplings:

$$
c_{1}^{t}=\frac{-2 e}{64 \pi^{2} m_{\eta}^{2}} \sum_{\ell} R_{\ell}^{t}\left[\frac{1}{2}+\frac{2}{3} \ln \left(\frac{m_{\ell}^{2}}{m_{\eta}^{2}}\right)\right], \quad c_{2}^{t}=\frac{-2 e}{64 \pi^{2} m_{\eta}^{2}} \frac{1}{4} \sum_{\ell} R_{\ell}^{t},
$$


where $R_{\ell}^{t}=\operatorname{Re}\left(h_{\ell 1} h_{\ell 2}^{*}\right)=h_{L} h_{S}$. Note that the formulas in eqs. (5.6) and (5.7) only differs a factor 2. Nevertheless, at the amplitude level, such a difference would be compensated when differentiating the two identical Majorana particle fields in the component-conserving effective operators $\mathcal{O}_{1 i}^{c}$ and $\mathcal{O}_{2 i}^{c}$.

With the above effective operators, the two DM particles couple to the charge and magnetic dipole moment of the nucleus, which give the spin-independent and spin-dependent differential cross sections

$$
\frac{d \sigma_{T}^{E}}{d E_{R}}=\frac{1}{2}\left[\left(c_{11}^{c}-2 c_{21}^{c}\right)^{2}+\left(c_{12}^{c}-2 c_{22}^{c}\right)^{2}+\frac{2}{4}\left(c_{1}^{t}-2 c_{2}^{t}\right)^{2}\right] e^{2} Z^{2} \frac{m_{T}}{4 \pi}\left(2-\frac{m_{T} E_{R}}{\mu_{N T}^{2} v^{2}}\right) F_{E}^{2}\left(q^{2}\right),
$$

and

$$
\frac{d \sigma_{T}^{M}}{d E_{R}}=\frac{1}{2}\left[\left(c_{11}^{c}-2 c_{21}^{c}\right)^{2}+\left(c_{12}^{c}-2 c_{22}^{c}\right)^{2}+\frac{2}{4}\left(c_{1}^{t}-2 c_{2}^{t}\right)^{2}\right] e^{2} \frac{1}{2 \pi} \frac{E_{R}}{v^{2}} \frac{m_{T}^{2} \lambda_{T}^{2}}{m_{N}^{2} \lambda_{N}^{2}} \frac{J_{T}+1}{3 J_{T}} F_{M}^{2}\left(q^{2}\right),
$$

where $\lambda_{N(T)}=e /\left(2 m_{N(T)}\right)$ is the magneton of the nucleon (target nucleus), $m_{N(T)}$ is the nucleon (target nucleus) mass, $J_{T}(Z)$ is the spin (charge) of the target nucleus, and $F_{E(M)}\left(q^{2}\right)$ is the form factor of the nucleus charge (magnetic dipole moment), respectively. Note that in eqs. (5.8) and (5.9), the factor $1 / 2$ accounts for the half density carried by each DM component in the Universe, while $2 / 4$ the right normalization of the transition scattering in accord with the previous two component-conserving ones.

From eqs. (5.8) and (5.9), it is obvious that the spin-independent part is the dominant one due to the large charge $Z^{2}$ enhancement for the heavy target nuclei, such as Xenon used in the LUX experiments. However, the differential cross section in the recoil energy $E_{R}$ is suppressed by an additional power of $v^{2}$ or $E_{R}$ compared with the usual Dirac fermionic DM case, resulting in a weak direct detection constraint. An estimate made in ref. [70] for ${ }_{54}^{129} \mathrm{Xe}$ with the typical reference recoiled energy and the similar masses of $\chi_{1,2}$ and $\eta$ gives the DM-nucleon scattering cross section of $\mathcal{O}\left(10^{-49}\right) \mathrm{cm}^{2}$, which is evidently much smaller than the current LUX bound for the spin-independent DM-nucleon cross section [69]. Therefore, we do not show the direct DM detection constraints on our model.

Finally, we point out that if the restriction on the real Yukawa couplings is relaxed, the same one-loop Feynman diagrams in figure 1 would also induce the following transition operators:

$$
\mathcal{O}_{3}^{t}=\overline{\chi_{2}} \gamma^{\mu} \partial^{\nu} \chi_{1} F_{\mu \nu}+\text { h.c., } \quad \mathcal{O}_{4}^{t}=-i \overline{\chi_{2}} \gamma^{\mu} \gamma^{5} \partial^{\nu} \chi_{1} F^{\alpha \beta} \epsilon_{\mu \nu \alpha \beta}+\text { h.c., }
$$

with the corresponding Wilson coefficients

$$
c_{3}^{t}=\frac{-2 e}{64 \pi^{2} m_{\eta}^{2}} \sum_{\ell} I_{\ell}^{t}\left[\frac{1}{2}+\frac{2}{3} \ln \left(\frac{m_{\ell}^{2}}{m_{\eta}^{2}}\right)\right], \quad c_{4}^{t}=\frac{-2 e}{64 \pi^{2} m_{\eta}^{2}} \frac{1}{4} \sum_{\ell} I_{\ell}^{t},
$$

where $I_{\ell}^{t}=\operatorname{Im}\left(h_{\ell 1} h_{\ell 2}^{*}\right)$. If the imaginary part of $h_{\ell i}$ is the same order as the real one, the dominant contribution to the direct detection experiments is given by the spin-independent cross section induced by the operator $\mathcal{O}_{3}^{t}$. In this case, there is no suppression caused by the small recoiled energy $E_{R}$ or the DM velocity $v^{2}$, and in turn the LUX experiments 
have already given strong constraints to this model, effectively ruling out all the parameter space trying to explain the X-ray excess.

\subsection{Collider searches}

A large portion of the parameter space of the present model has already been probed by the LEP and LHC experiments. Since our two DM components do not directly couple to the electron at tree-level by the construction, they cannot be directly pair produced at the LEP so that the relevant monophoton constraints [77] do not apply.

Another collider signal in our model is related to the production of the singly-charged scalar $\eta$ mediated by a virtual photon or $Z$-boson. When the mass of $\eta$ is lighter than about $100 \mathrm{GeV}$, it can be pair produced at the $e^{+} e^{-}$colliders, which has been already ruled out by the null results at the LEP. Since the maximum energy of the LEP is about $200 \mathrm{GeV}$, we take $100 \mathrm{GeV}$ as the LEP bound for the charged mediator mass as an illustration in our study. At the LHC, the signal of this leptophilic model is the creation of the charged mediator pair via the Drell-Yan process, each of which decays further into a lepton plus one of the DM particles [70, 72, 73]. The signature at hadron colliders is a pair of opposite-sign leptons with some missing transverse energy. Such a signature is very similar to that of searching for the right-handed sleptons with mixings in the MSSM at the colliders, for which the current LHC bound of the mass of the charged scalar is about $240 \mathrm{GeV}$ as presented in figure $8 \mathrm{a}$ in ref. [78], which is more stringent than the LEP direct search bound.

In this work, we use both LEP and LHC bounds in our numerical studies, which can be transformed into the mass bounds of the two nearly degenerate DM components for a given mass ratio $R_{M}=m_{\eta} / m_{\chi}$. In figure 3 , the excluded regions for both experimental bounds are plotted as the purple and yellow regions bounded by the dot-dashed and dotted lines. Note that the latter LHC constraint can be relaxed a lot for the present model. The LHC bound of $240 \mathrm{GeV}$ for the charged scalar $\eta$ is the most stringent one in figure $8 \mathrm{a}$ in ref. [78]. The actual restricted region in the $m_{\eta}-m_{\chi}$ plane is much irregular, and the constraint becomes more loose when the DM mass approaches the mediator one due to the decreasing of the missing energy. In figure 4 , we show the actual LHC bound on the $m_{\eta}-m_{\chi}$ plane given in ref. [78], together with the allowed X-ray signal region and other constraints with the Yukawa couplings $\left(h_{S}, h_{L}\right)=(0.113,1.13)$. Another important issue is the assumption made in the analysis of ref. [78] that the decay of the charged scalar is only to the first two generations of the charged leptons plus a large missing energy. Nevertheless, in our model, $\eta$ can only have about one fourth probability to $\mu^{+}+\mu^{-}$, whereas the other $3 / 4$ of the decay branching ratio related to the $\tau$ lepton cannot be probed. As the detailed analysis of the LHC bound on our model is beyond the scope of the present paper, we are content with the most stringent bound in ref. [78] in our exploration of the model parameter space.

\section{Final results}

Our results on the parameter space are shown in figure 3 for different choices of the mass ratios $R_{M}=m_{\eta} / m_{\chi}$ and the Yukawa coupling ratios $R_{h}=h_{S} / h_{L}$. The orange region with the thin black boundaries represents the parameter space which can be used to explain the 


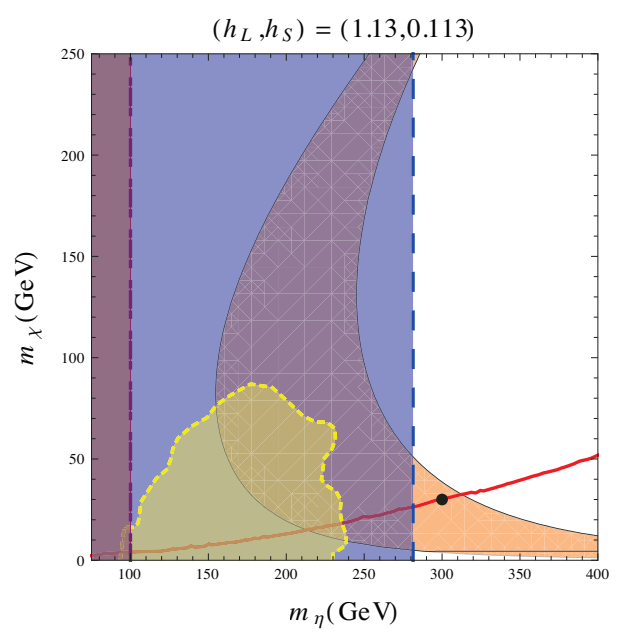

Figure 4. Label is the same as figure 3 but in the $m_{\eta}-m_{\chi}$ plane for $\left(h_{L}, h_{S}\right)=(1.13,0.113)$. The black point represents the benchmark point given in the context.

X-ray line fluxes, while the thick red curve gives the correct DM relic abundance. The blue, yellow and purple regions bounded by the dashed, dotted and dot-dashed lines are excluded by the constraints from the $\tau \rightarrow \mu \gamma$ process, LEP and LHC, respectively. A further constraint is originated from the perturbativity requirement of the large Yukawa coupling $h_{L}$, which is assumed to be less than 3 .

In figure 3 , we arrange the diagrams in rows and columns in terms of the mass ratios $R_{M}$ and the coupling ratios $R_{h}$ in order to show the trend of how the allowed parameter space changes when these two parameters vary. It is seen that there are still parameter spaces in which the present two-component DM model can explain the cluster X-ray line and DM relic abundance in the Universe, simultaneously, despite of the very strong constraints from flavor and collider physics. As an illustration, we present a prototypical benchmark point: $h_{S}=0.113, h_{L}=1.13, m_{\chi}=30 \mathrm{GeV}$ and $m_{\eta}=300 \mathrm{GeV}$. Figure 4 illustrates the parameter space in the $m_{\eta}-m_{\chi}$ plane with the fixed Yukawa couplings $\left(h_{L}, h_{S}\right)=(1.13,0.113)$, in which the current ATLAS bounds on $m_{\eta}$ and $m_{\chi}$ are shown more clearly.

\section{Conclusions and discussions}

We have proposed a two-component DM model to understand the recent unidentified Xray line from the observation of the Andromeda galaxy and the distant galaxy clusters. In order for the two DM particles $\chi_{1,2}$ to only couple to the right-handed $\mu$ and $\tau$ via the Yukawa couplings, a new singly-charged scalar mediator $\eta$ is introduced. Both $\chi_{1,2}$ and $\eta$ are odd under the dark $Z_{2}$ symmetry. In spite of the very stringent constraints from flavor physics, direct DM searches and collider bounds from the LEP and LHC, we have found some parameter space which can explain the X-ray line with the correct DM relic abundance.

As seen from eq. (5), the successful explanation of the cluster X-ray flux depends on the $3.5 \mathrm{keV}$ mass splitting $\Delta m$ between the two DM components, which is naturally motivated 
by the measured energy of the X-ray line. The next question is to investigate the stability of such a small splitting against the radiative corrections, especially when we notice the DM Majorana masses are of $\mathcal{O}(10 \mathrm{GeV})$. However, such a worry can be avoided for the present DM model. Since the leading order radiative correction to the DM Majorana masses appears at three-loop level, we find that the correction to the mass splitting is always of order sub-keV, which is achieved by the suppressions from the loop factors and the various couplings. Therefore, we expect that the $\mathrm{keV}$ order of the DM mass splitting is naturally guaranteed against the radiative corrections in our model.

In our study, we have used the most stringent bound from the ATLAS [78] to constrain the charged scalar mediator mass $m_{\eta}$. However, this constraint can be relaxed if we take into account the difference of the present model from the assumptions made in ref. [78]. This analysis is helpful because this constraint excludes all or part of the signal region in some parameter spaces, such as those shown in the second and third graphs in the first row of figure 3. A detailed investigation of this collider constraint may reopen these parameter windows, which will be worth of a future work.

\section{Acknowledgments}

The work was supported in part by National Center for Theoretical Sciences, National Science Council (NSC-101-2112-M-007-006-MY3) and National Tsing Hua University (Grant Nos. 103N1087E1 and 103N2724E1).

Open Access. This article is distributed under the terms of the Creative Commons Attribution License (CC-BY 4.0), which permits any use, distribution and reproduction in any medium, provided the original author(s) and source are credited.

\section{References}

[1] G. Bertone, D. Hooper and J. Silk, Particle dark matter: Evidence, candidates and constraints, Phys. Rept. 405 (2005) 279 [hep-ph/0404175] [INSPIRE].

[2] K. Freese, M. Lisanti and C. Savage, Annual Modulation of Dark Matter: A Review, Rev. Mod. Phys. 85 (2013) 1561 [arXiv: 1209.3339] [InSPIRE].

[3] G. Jungman, M. Kamionkowski and K. Griest, Supersymmetric dark matter, Phys. Rept. 267 (1996) 195 [hep-ph/9506380] [INSPIRE].

[4] P.F. Smith and J.D. Lewin, Dark Matter Detection, Phys. Rept. 187 (1990) 203 [InSPIRE].

[5] M. Cirelli, Indirect Searches for Dark Matter: a status review, Pramana 79 (2012) 1021 [arXiv: 1202.1454] [INSPIRE].

[6] E. Bulbul, M. Markevitch, A. Foster, R.K. Smith, M. Loewenstein and S.W. Randall, Detection of An Unidentified Emission Line in the Stacked X-ray spectrum of Galaxy Clusters, Astrophys. J. 789 (2014) 13 [arXiv:1402.2301] [INSPIRE].

[7] A. Boyarsky, O. Ruchayskiy, D. Iakubovskyi and J. Franse, An unidentified line in X-ray spectra of the Andromeda galaxy and Perseus galaxy cluster, arXiv:1402.4119 [INSPIRE]. 
[8] H. Ishida, K.S. Jeong and F. Takahashi, $7 \mathrm{keV}$ sterile neutrino dark matter from split flavor mechanism, Phys. Lett. B 732 (2014) 196 [arXiv: 1402.5837] [INSPIRE].

[9] D.P. Finkbeiner and N. Weiner, An X-Ray Line from eXciting Dark Matter, arXiv: 1402.6671 [INSPIRE].

[10] T. Higaki, K.S. Jeong and F. Takahashi, The $7 \mathrm{keV}$ axion dark matter and the X-ray line signal, Phys. Lett. B 733 (2014) 25 [arXiv:1402.6965] [INSPIRE].

[11] J. Jaeckel, J. Redondo and A. Ringwald, A $3.55 \mathrm{keV}$ hint for decaying axion-like particle dark matter, Phys. Rev. D 89 (2014) 103511 [arXiv:1402.7335] [INSPIRE].

[12] M. Czerny, T. Higaki and F. Takahashi, Multi-Natural Inflation in Supergravity, JHEP 05 (2014) 144 [arXiv:1403.0410] [INSPIRE].

[13] H.M. Lee, S.C. Park and W.-I. Park, Cluster X-ray line at $3.5 \mathrm{keV}$ from axion-like dark matter, arXiv: 1403.0865 [INSPIRE].

[14] K.N. Abazajian, Resonantly-Produced 7keV Sterile Neutrino Dark Matter Models and the Properties of Milky Way Satellites, Phys. Rev. Lett. 112 (2014) 161303 [arXiv:1403.0954] [INSPIRE].

[15] R. Krall, M. Reece and T. Roxlo, Effective field theory and keV lines from dark matter, arXiv: 1403.1240 [INSPIRE].

[16] C.E. Aisati, T. Hambye and T. Scarna, Can a millicharged dark matter particle emit an observable gamma-ray line?, arXiv:1403.1280 [INSPIRE].

[17] K. Hamaguchi, M. Ibe, T.T. Yanagida and N. Yokozaki, Testing the Minimal Direct Gauge Mediation at the LHC, arXiv:1403.1398 [INSPIRE].

[18] J.-C. Park, S.C. Park and K. Kong, X-ray line signal from $7 \mathrm{keV}$ axino dark matter decay, Phys. Lett. B 733 (2014) 217 [arXiv: 1403.1536] [INSPIRE].

[19] M.T. Frandsen, F. Sannino, I.M. Shoemaker and O. Svendsen, X-ray Lines from Dark Matter: The Good, The Bad and The Unlikely, JCAP 05 (2014) 033 [arXiv:1403.1570] [INSPIRE].

[20] S. Baek and H. Okada, 7 keV Dark Matter as X-ray Line Signal in Radiative Neutrino Model, arXiv:1403.1710 [INSPIRE].

[21] K. Nakayama, F. Takahashi and T.T. Yanagida, The $3.5 \mathrm{keV}$ X-ray line signal from decaying moduli with low cutoff scale, Phys. Lett. B 735 (2014) 338 [arXiv:1403.1733] [INSPIRE].

[22] K.-Y. Choi and O. Seto, X-ray line signal from decaying axino warm dark matter, Phys. Lett. B 735 (2014) 92 [arXiv:1403.1782] [INSPIRE].

[23] M. Cicoli, J.P. Conlon, M.C.D. Marsh and M. Rummel, A $3.55 \mathrm{keV}$ Photon Line and its Morphology from a $3.55 \mathrm{keV}$ ALP Line, Phys. Rev. D 90 (2014) 023540 [arXiv:1403.2370] [INSPIRE].

[24] F. Bezrukov and D. Gorbunov, Relic Gravity Waves and 7keV Dark Matter from a GeV scale inflaton, arXiv:1403.4638 [INSPIRE].

[25] R. Allahverdi, B. Dutta and Y. Gao, keV Photon Emission from Light Nonthermal Dark Matter, Phys. Rev. D 89 (2014) 127305 [arXiv: 1403.5717] [INSPIRE].

[26] T. Tsuyuki, Neutrino masses, leptogenesis and sterile neutrino dark matter, Phys. Rev. D 90 (2014) 013007 [arXiv: 1403.5053] [INSPIRE]. 
[27] C. Kolda and J. Unwin, X-ray lines from R-parity violating decays of keV sparticles, Phys. Rev. D 90 (2014) 023535 [arXiv: 1403.5580] [INSPIRE].

[28] A.G. Dias, A.C.B. Machado, C.C. Nishi, A. Ringwald and P. Vaudrevange, The Quest for an Intermediate-Scale Accidental Axion and Further ALPs, JHEP 06 (2014) 037 [arXiv:1403.5760] [INSPIRE].

[29] N.E. Bomark and L. Roszkowski, $3.5 \mathrm{keV}$ x-ray line from decaying gravitino dark matter, Phys. Rev. D 90 (2014) 011701(R) [arXiv:1403.6503] [INSPIRE].

[30] S.P. Liew, Axino dark matter in light of an anomalous X-ray line, JCAP 05 (2014) 044 [arXiv: 1403.6621] [INSPIRE].

[31] K. Nakayama, F. Takahashi and T.T. Yanagida, Anomaly-free flavor models for Nambu-Goldstone bosons and the $3.5 \mathrm{keV}$ X-ray line signal, Phys. Lett. B 734 (2014) 178 [arXiv: 1403.7390] [INSPIRE].

[32] Z. Kang, P. Ko, T. Li and Y. Liu, Natural X-ray Lines from the Low Scale Supersymmetry Breaking, arXiv:1403.7742 [INSPIRE].

[33] H. Okada, Two loop Induced Dirac Neutrino Model and Dark Matters with Global U(1)' Symmetry, arXiv:1404.0280 [INSPIRE].

[34] B.R. Safdi, M. Lisanti, J. Spitz and J.A. Formaggio, Annual Modulation of Cosmic Relic Neutrinos, Phys. Rev. D 90 (2014) 043001 [arXiv:1404.0680] [inSPIRE].

[35] S.V. Demidov and D.S. Gorbunov, SUSY in the sky or keV signature of sub-GeV gravitino dark matter, arXiv:1404.1339 [INSPIRE].

[36] F.S. Queiroz and K. Sinha, The Poker Face of the Majoron Dark Matter Model: LUX to keV Line, Phys. Lett. B 735 (2014) 69 [arXiv:1404.1400] [INSPIRE].

[37] E. Dudas, L. Heurtier and Y. Mambrini, Generating X-ray lines from annihilating dark matter, arXiv:1404.1927 [INSPIRE].

[38] K.P. Modak, $3.5 \mathrm{keV} \mathrm{X-ray} \mathrm{Line} \mathrm{Signal} \mathrm{from} \mathrm{Decay} \mathrm{of} \mathrm{Right-Handed} \mathrm{Neutrino} \mathrm{due} \mathrm{to}$ Transition Magnetic Moment, arXiv:1404.3676 [INSPIRE].

[39] J.M. Cline, Y. Farzan, Z. Liu, G.D. Moore and W. Xue, $3.5 \mathrm{keV} X$-rays as the "21 cm line" of dark atoms and a link to light sterile neutrinos, Phys. Rev. D 89 (2014) 121302(R) [arXiv: 1404.3729] [INSPIRE].

[40] H. Okada and T. Toma, The $3.55 \mathrm{keV}$ X-ray Line Signal from Excited Dark Matter in Radiative Neutrino Model, arXiv:1404.4795 [INSPIRE].

[41] J.L. Rosner, Three sterile neutrinos in E6, arXiv:1404.5198 [INSPIRE].

[42] H.M. Lee, Magnetic dark matter for the X-ray line at $3.55 \mathrm{keV}$, arXiv:1404.5446 [INSPIRE].

[43] D.J. Robinson and Y. Tsai, A Dynamical Framework for KeV Dirac Neutrino Warm Dark Matter, arXiv:1404.7118 [INSPIRE].

[44] J.P. Conlon and F.V. Day, $3.55 \mathrm{keV}$ photon lines from axion to photon conversion in the Milky Way and M31, arXiv:1404.7741 [INSPIRE].

[45] K.S. Babu and R.N. Mohapatra, $7 \mathrm{keV}$ Scalar Dark Matter and the Anomalous Galactic X-ray Spectrum, Phys. Rev. D 89 (2014) 115011 [arXiv: 1404.2220] [INSPIRE].

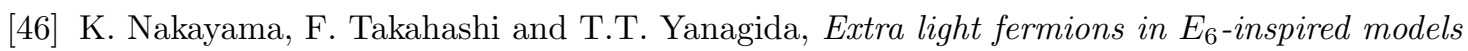
and the $3.5 \mathrm{keV} X$-ray line signal, arXiv: 1405.4670 [INSPIRE]. 
[47] S. Baek, P. Ko and W.-I. Park, The $3.5 \mathrm{keV} \mathrm{X-ray} \mathrm{line} \mathrm{signature} \mathrm{from} \mathrm{annihilating} \mathrm{and}$ decaying dark matter in Weinberg model, arXiv:1405.3730 [INSPIRE].

[48] S. Chakraborty, D.K. Ghosh and S. Roy, 7 keV Sterile neutrino dark matter in $\mathrm{U}(1)_{R}$-lepton number model, arXiv:1405.6967 [INSPIRE].

[49] M. Kawasaki, N. Kitajima and F. Takahashi, Relaxing Isocurvature Bounds on String Axion Dark Matter, arXiv:1406.0660 [INSPIRE].

[50] N. Chen, Z. Liu and P. Nath, $3.5 \mathrm{keV}$ Galactic Emission Line as a Signal from the Hidden Sector, arXiv: 1406.0687 [INSPIRE].

[51] J.P. Conlon and A.J. Powell, A $3.55 \mathrm{keV}$ line from $D M \rightarrow a \rightarrow \gamma$ : predictions for cool-core and non-cool-core clusters, arXiv:1406.5518 [INSPIRE].

[52] H. Ishida and H. Okada, $3.55 \mathrm{keV} \mathrm{X-ray} \mathrm{Line} \mathrm{Interpretation} \mathrm{in} \mathrm{Radiative} \mathrm{Neutrino} \mathrm{Model,}$ arXiv: 1406.5808 [INSPIRE].

[53] J. Fan, A. Katz, L. Randall and M. Reece, Double-Disk Dark Matter, Phys. Dark Univ. 2 (2013) 139 [arXiv: 1303.1521] [INSPIRE].

[54] J. Fan, A. Katz, L. Randall and M. Reece, Dark-Disk Universe, Phys. Rev. Lett. 110 (2013) 211302 [arXiv: 1303.3271] [INSPIRE].

[55] M. McCullough and L. Randall, Exothermic Double-Disk Dark Matter, JCAP 10 (2013) 058 [arXiv: 1307.4095] [INSPIRE].

[56] C.-Q. Geng, D. Huang and L.-H. Tsai, Imprint of Multi-component Dark Matter on AMS-02, Phys. Rev. D 89 (2014) 055021 [arXiv: 1312.0366] [INSPIRE].

[57] C.-Q. Geng, D. Huang and L.-H. Tsai, Cosmic Ray Excesses from Multi-component Dark Matter Decays, arXiv:1405.7759 [INSPIRE].

[58] A.G. Doroshkevich and M.Yu. Khlopov, On The Physical Nature Of Hidden Mass In The Universe, Sov. J. Nucl. Phys. 39 (1984) 551.

[59] M.Yu. Khlopov, Physical arguments, favouring multicomponent dark matter, in 30th Rencontres de Moriond: Perspectives in Particle Physics, Atomic Physics and Gravitation, B. Guiderdoni, G. Greene, D. Hinds and J. Tran Thanh Van eds., Editions Frontieres, Gif-sur-Yvette France (1995), pg. 133 [INSPIRE].

[60] M.Yu. Khlopov and C. Kouvaris, Composite dark matter from a model with composite Higgs boson, Phys. Rev. D 78 (2008) 065040 [arXiv:0806.1191] [INSPIRE].

[61] K. Griest and D. Seckel, Three exceptions in the calculation of relic abundances, Phys. Rev. D 43 (1991) 3191 [INSPIRE].

[62] E. Kolb and M. Turner, The Early Universe, Westview Press, U.S.A. (1994).

[63] N. Arkani-Hamed, A. Delgado and G.F. Giudice, The Well-tempered neutralino, Nucl. Phys. B 741 (2006) 108 [hep-ph/0601041] [INSPIRE].

[64] M. Srednicki, R. Watkins and K.A. Olive, Calculations of Relic Densities in the Early Universe, Nucl. Phys. B 310 (1988) 693 [INSPIRE].

[65] K.A. Olive, D.N. Schramm and G. Steigman, Limits on New Superweakly Interacting Particles from Primordial Nucleosynthesis, Nucl. Phys. B 180 (1981) 497 [INSPIRE].

[66] Planck collaboration, P.A.R. Ade et al., Planck 2013 results. I. Overview of products and scientific results, arXiv:1303.5062 [INSPIRE]. 
[67] D. Schmidt, T. Schwetz and T. Toma, Direct Detection of Leptophilic Dark Matter in a Model with Radiative Neutrino Masses, Phys. Rev. D 85 (2012) 073009 [arXiv:1201.0906] [INSPIRE].

[68] Particle Data Group collaboration, J. Beringer et al., Review of Particle Physics (RPP), Phys. Rev. D 86 (2012) 010001 [inSPIRE].

[69] LUX collaboration, D.S. Akerib et al., First results from the LUX dark matter experiment at the Sanford Underground Research Facility, Phys. Rev. Lett. 112 (2014) 091303 [arXiv: 1310.8214] [INSPIRE].

[70] Y. Bai and J. Berger, Lepton Portal Dark Matter, arXiv:1402.6696 [INSPIRE].

[71] P. Agrawal, S. Blanchet, Z. Chacko and C. Kilic, Flavored Dark Matter and Its Implications for Direct Detection and Colliders, Phys. Rev. D 86 (2012) 055002 [arXiv:1109.3516] [INSPIRE].

[72] P. Agrawal, Z. Chacko and C.B. Verhaaren, Leptophilic Dark Matter and the Anomalous Magnetic Moment of the Muon, arXiv:1402.7369 [INSPIRE].

[73] S. Chang, R. Edezhath, J. Hutchinson and M. Luty, Leptophilic Effective WIMPs, Phys. Rev. D 90 (2014) 015011 [arXiv: 1402.7358] [INSPIRE].

[74] A.L. Fitzpatrick and K.M. Zurek, Dark Moments and the DAMA-CoGeNT Puzzle, Phys. Rev. D 82 (2010) 075004 [arXiv: 1007.5325] [INSPIRE].

[75] C.M. Ho and R.J. Scherrer, Anapole Dark Matter, Phys. Lett. B 722 (2013) 341 [arXiv: 1211.0503] [INSPIRE].

[76] E. Del Nobile, G.B. Gelmini, P. Gondolo and J.-H. Huh, Direct detection of Light Anapole and Magnetic Dipole DM, JCAP 06 (2014) 002 [arXiv:1401.4508] [INSPIRE].

[77] P.J. Fox, R. Harnik, J. Kopp and Y. Tsai, LEP Shines Light on Dark Matter, Phys. Rev. D 84 (2011) 014028 [arXiv: 1103.0240] [INSPIRE].

[78] ATLAS collaboration, Search for direct-slepton and direct-chargino production in final states with two opposite-sign leptons, missing transverse momentum and no jets in $20 / \mathrm{fb}$ of $p p$ collisions at $\sqrt{s}=8 \mathrm{TeV}$ with the ATLAS detector, CERN, Geneva, 2013,

ATLAS-CONF-2013-049. 